Check for updates

Cite this: RSC Adv., 2018, 8, 9253

\title{
Internalization, cytotoxicity, oxidative stress and inflammation of multi-walled carbon nanotubes in human endothelial cells: influence of pre- incubation with bovine serum albumin
}

\author{
Jimin Long, $\dagger^{\mathrm{abc}}$ Xianqiang $\mathrm{Li}, \dagger^{\mathrm{c}}$ Yang Kang, (ID a Yanhuai Ding, (D) *b Zhipeng Gu (DD *a \\ and $\mathrm{Yi} \mathrm{CaO}^{\mathrm{b}}$
}

When entering circulation, multi-walled carbon nanotubes (MWCNTs) will inevitably adsorb proteins, which can consequently influence their toxicity to cells lining human blood vessels. In this study, we investigated the influence of pre-incubation with bovine serum albumin (BSA) on internalization, cytotoxicity, oxidative stress and inflammation induced by pristine/carboxylated MWCNTs to human umbilical vein endothelial cells (HUVECs). Atomic force microscopy (AFM) indicated the adsorption of proteins onto the surface of MWCNTs, which consequently increased the diameter. Pre-incubation with BSA did not obviously influence the hydrodynamic sizes, but decreased the zeta potential of MWCNTs. Transmission electron microscopy (TEM) indicated the internalization of both types of MWCNTs into HUVECs, whereas preincubation with BSA appeared to enhance the internalization. MWCNT exposure induced cytotoxicity and oxidative stress, as well as a modest inflammatory response shown as an increased THP-1 adhesion to HUVECs, but not release of interleukin 6 (IL-6) or tumor necrosis factor (TNF $\alpha$ ). Exposure to MWCNTs pre-incubated with BSA induced less cytotoxicity to HUVECs, associated with increased intracellular glutathione (GSH). However, MWCNTs induced IL-6 and TNF $\alpha$ release, as well as THP-1 adhesion to HUVECS, were enhanced after pre-incubation with BSA. In summary, these data indicated that preincubation with BSA could enhance the internalization of MWCNTs to HUVECs, which consequently reduces the cytotoxicity and oxidative stress, but enhances the inflammatory response of MWCNTs. The reduced cytotoxicity and oxidative stress, and enhanced inflammatory responses are likely due to a combined effect of BSA and MWCNTs, which suggests that when assessing the biological effects of MWCNTs in circulation, it is necessary to consider the interactions between MWCNTs and serum proteins.

Received 15th January 2018 Accepted 22nd February 2018

DOI: $10.1039 / \mathrm{c} 8 \mathrm{ra00445e}$

rsc.li/rsc-advances health effects of MWCNTs to ensure their safe use. In particular, the toxicity of MWCNTs to the vascular system should be carefully assessed, since the vascular system is required to deliver NPs to realize their medicinal purpose. ${ }^{5,6}$ Indeed, there have been reports showing the adverse health effects of MWCNTs to the vascular system, such as induction of oxidative stress, ${ }^{7}$ promotion of endothelial activation ${ }^{8}$ and acceleration of foam cell formation. ${ }^{9}$ Still, more work is needed to investigate the interactions between MWCNTs and the vascular system both in vivo and in vitro. ${ }^{\mathbf{1 0}}$

When entering a biological microenvironment, NPs could rapidly adsorb bio-molecules, which could critically influence the biological effects of NPs. One of the most studied examples is the adsorption of serum proteins, which has been shown to deeply influence the colloidal aspects of NPs, as well as the interactions between NPs and cells. ${ }^{\mathbf{1 1 , 1 2}}$ This is also the case for MWCNTs. ${ }^{13}$ For example, Cai et al. ${ }^{14}$ and Shannahan et al. ${ }^{15}$ revealed complex protein corona formation when MWCNTs were incubated with biological fluids, and the binding efficiency 
was dependent on the physicochemical properties of NPs. Zhao et al. $^{\mathbf{1 6}}$ found diameter-dependent adsorption of bovine serum albumin (BSA) to MWCNTs, and the formation of protein corona consequently reduced the cytotoxicity of MWCNTs to astrocytes. In another study, Bai et $a .^{17}$ showed that the adsorption of ovalbumin (OVA) to MWCNTs promoted macrophage activation, which indicated that MWCNTs pre-incubated with BSA could be used in the delivery of antigens. However, relatively few studies investigated the influence of protein corona formation on the toxicity of MWCNTs to cells lining blood vessels. De Paoli et al. ${ }^{18}$ showed that the adsorption of different plasma proteins to MWCNTs greatly influenced the interactions between MWCNTs and human blood platelets. However, more studies are needed to understand the influence of serum proteins on MWCNT induced toxicity to other types of cells in blood vessels.

The lumen of human blood vessels is covered by a thin layer of endothelial cells (ECs), which are the first contact for NPs entering circulation. Thus, it has been suggested that the interactions between NPs and ECs should be carefully assessed, even though only some of NPs were aimed at targeting ECs. ${ }^{5,6}$ Moreover, ECs play a pivotal role in the regulation of blood vessel function, and assessment of the toxicity of NPs to ECs may provide important information about the development of vascular diseases, such as atherosclerosis., ${ }^{5,9}$ In this study, human umbilical vein endothelial cells (HUVECs) were used as the in vitro model for ECs, and the influence of pre-incubation with BSA on the interactions between MWCNTs and HUVECs was studied. Pristine and carboxylated MWCNTs were used, because it has been suggested that the surface chemistry of NPs could influence NP-protein interactions., ${ }^{5,11}$ Atomic force microscope (AFM) was used to indicate the change of diameter of the MWCNTs due to the pre-incubation of BSA. Moreover, the change of hydrodynamic sizes and zeta potential of MWCNTs due to the incubation with BSA were also measured. To indicate the influence of BSA pre-incubation on MWCNT-HUVEC interactions, the internalization, cytotoxicity, oxidative stress, release of inflammatory cytokines and monocyte adhesion to HUVECs induced by MWCNTs, with or without pre-incubation with BSA, was investigated.

\section{Materials and methods}

\subsection{Cell culture}

HUVECs (passage 1) were purchased from ScienCell Research Laboratories (Carlsbad, CA) and cultured in supplemented endothelial cell medium (ECM) as we described previously. ${ }^{19}$ THP-1 monocytes (ATCC) were cultured in supplemented RPMI1640 medium as we previously described. ${ }^{20}$

\subsection{MWCNT preparation}

The pristine MWCNTs (code: XFM19) and carboxylated MWCNTs (code: XFM21) were purchased from Nanjing XFNANO Materials Tech Co., Ltd (Nanjing, China). The physicochemical properties of XFM19 and XFM21 from the supplier are summarized in Table 1. To make the suspension of
MWCNTs for cell exposure, a stock solution of $1.28 \mathrm{mg} \mathrm{mL}$ particles in Milli-Q water containing $1 \mathrm{mg} \mathrm{mL}^{-1}$ BSA (SigmaAldrich, USA) was prepared by sonication for 8 min twice with continuous cooling on ice using an ultrasonic processor FS$250 \mathrm{~N}$ (20\% amplitude; Shanghai Shengxi, China). After sonication, the stock solution was diluted to $640 \mu \mathrm{g} \mathrm{mL}{ }^{-1}$ with or without the presence of 5\% BSA. After incubation for $30 \mathrm{~min}$, the MWCNT suspensions were then diluted to $640 \mu \mathrm{g} \mathrm{mL}^{-1}$ in serum free medium for exposure. The control cells were incubated with an equal amount of vehicles in serum free medium.

\subsection{In situ AFM}

The MWCNT suspensions were prepared as $640 \mu \mathrm{g} \mathrm{mL} \mathrm{m}^{-1}$ and incubated with Milli-Q water or 5\% BSA for at least $30 \mathrm{~min}$. After that, the MWCNT suspensions were diluted ten times in water, and in situ AFM experiments were performed on a Bruker MultiMode 8 by using the Peakforce mode at room temperature. The samples were prepared by depositing a drop of the diluted aqueous dispersion on a newly cleaved mica surface. A ScanAsyst-Fluid probe with a nominal spring constant of $0.7 \mathrm{~N} \mathrm{~m}^{-1}$ and a nominal tip radius $R=20 \mathrm{~nm}$ was used in all the tests (ScanAsyst Fluid+, Bruker AXS). Nanoscope analysis 1.8 software was employed to analyze the topographic images. A total of 20 randomly selected MWCNTs in each sample were analyzed, and the changes of diameters due to the incubation with BSA were calculated.

\subsection{Hydrodynamic size and zeta potential}

The MWCNT suspensions were prepared as indicated above, and then diluted to $16 \mu \mathrm{g} \mathrm{mL}{ }^{-1}$ in Milli-Q water or $0.125 \%$ BSA. The hydrodynamic size distribution and zeta potential were measured using a Zetasizer Nano ZS90 (Malvern, UK).

\subsection{Transmission electron microscopy (TEM)}

TEM was used to indicate the internalization of MWCNTs. Briefly, cells were seeded at $5 \times 10^{5}$ on $60 \mathrm{~mm}$ diameter cell culture Petri dishes and grown for 2 days before exposure. The MWCNT suspensions were prepared as indicated above, and the cells were exposed to $64 \mu \mathrm{g} \mathrm{mL}{ }^{-1}$ XFM19 or XFM21, with or without pre-incubation with BSA in serum free medium. After $6 \mathrm{~h}$ exposure, the cells were rinsed once and then scratched by using a cell scraper. After centrifuge, the cells were fixed with $2.5 \%$ glutaraldehyde in PBS overnight, post-fixed with $1 \% \mathrm{OsO}_{4}$ for $3 \mathrm{~h}$, dehydrated in a graded series of ethanol, and embedded in epoxy Resin (Epon 812). The samples were then sectioned using an ultramicrotome at $70 \mathrm{~nm}$, placed on carbon film supported by copper grids, stained with uranyl acetate and lead citrate, and observed under a TEM (JEM-1230, JEOL Ltd., Tokyo, Japan) operated at $80 \mathrm{kV}$.

\subsection{Cytotoxicity (CCK-8 and neutral red uptake assays)}

The cytotoxicity of MWCNTs to HUVECs was assessed by CCK-8 (cell counting kit-8) and neutral red uptake assays. CCK-8 has been developed as a substitute for the commonly-used MTT assay to reflect mitochondrial activity. ${ }^{19}$ Neutral red uptake 
Table 1 The physicochemical properties of XFM19 (pristine MWCNT) and XFM21 (carboxylated MWCNT) provided by the suppliers ${ }^{a}$

\begin{tabular}{lll}
\hline Code & XFM19 & XFM21 \\
\hline Purity & $>95 \%$ & $>95 \%$ \\
-COOH content & & $1.23 \mathrm{wt} \%$ \\
Primary size & OD: $20-30 \mathrm{~nm} ; \mathrm{ID}: 5-10 \mathrm{~nm} ; \mathrm{L}: 10-30 \mu \mathrm{M}$ & OD: $20-30 \mathrm{~nm}$; ID: $5-10 \mathrm{~nm} ; \mathrm{L}: 10-30 \mu \mathrm{M}$ \\
SSA & $>110 \mathrm{~m}^{2} \mathrm{~g}^{-1}$ & $>110 \mathrm{~m}^{2} \mathrm{~g}^{-1}$ \\
Tap density & $0.28 \mathrm{~g} \mathrm{~m}^{-3}$ & $0.28 \mathrm{~g} \mathrm{~m}^{-3}$ \\
True density & $\sim 2.1 \mathrm{~g} \mathrm{~cm}^{-3}$ & $\sim 2.1 \mathrm{~g} \mathrm{~cm}^{-3}$ \\
EC & $>100 \mathrm{~s} \mathrm{~cm}^{-1}$ & $>100 \mathrm{~s} \mathrm{~cm}^{-1}$
\end{tabular}

${ }^{a}$ Abbreviations: EC: electric conductivity; ID: inner diameter; L: length; OD: outer diameter; SSA: special surface area. ${ }^{b}$ The rate of the surface carbon atom was $8-10 \mathrm{~mol} \%$.

assay is a commonly used assay to reflect the damage sustained to lysosomes. ${ }^{21}$ Both of the assays were done by using commercial kits following the manufacturer's instructions (Beyotime, Nantong, China). Briefly, $2.0 \times 10^{4}$ per well HUVECs were seeded in 24-well plates and grown for 2 days before exposure. After that, the cells were incubated with $64 \mu \mathrm{g} \mathrm{mL} \mathrm{m}^{-1}$ XFM19 or XFM21 with or without pre-incubation with BSA in serum free medium. After incubation for $6 \mathrm{~h}$, the cells were rinsed once with Hanks' solution, and then the CCK-8 and neutral red uptake assays were done according to the manufacturer's instructions. The products were read by an ELISA reader (Synergy HT, BioTek, USA).

\subsection{Oxidative stress}

The oxidative stress in HUVECs was assessed by the measurement of intracellular glutathione (GSH) and ROS. The intracellular GSH was measured by using a fluorescence probe monochlorobimane (MCB; Sigma-Aldrich), which can be used to estimate GSH due to the formation of a fluorescent GSH-MCB adduct catalyzed by glutathione $S$-transferase. ${ }^{22}$ The intracellular ROS was measured using DCFH-DA, which can form a fluorescent product upon reaction with intracellular ROS. ${ }^{23}$ Both of the assays were performed as previously described. ${ }^{23,24}$ Briefly, $1 \times 10^{4}$ per well HUVECs were seeded on 96-well black plates and grown for 2 days prior to exposure. MWCNTs were prepared as indicated above, and the cells were incubated with $64 \mu \mathrm{g} \mathrm{mL} \mathrm{mL}^{-1}$ XFM19 or XFM21 with or without pre-incubation with BSA in serum free medium. After $6 \mathrm{~h}$ exposure, the cells were rinsed once, and then incubated with $50 \mu \mathrm{M}$ MCB or $10 \mu \mathrm{M}$ DCFH-DA in serum-free medium for $30 \mathrm{~min}^{23,24}$ After rinsing once again, the fluorescence was read at Ex $360 \pm 44 \mathrm{~nm}$ and Em $460 \pm 40 \mathrm{~nm}$ (for GSH), or Ex $485 \pm 20 \mathrm{~nm}$ and Em $528 \pm 20 \mathrm{~nm}$ (for ROS) by an ELISA reader.

\subsection{ELISA}

The supernatants from CCK-8 or neutral red uptake assays were collected. To free the interference from MWCNTs, the medium was centrifuged at $16000 \times g$ for $30 \mathrm{~min}$, and the supernatants were collected and stored at $-20{ }^{\circ} \mathrm{C}$ within one month before analysis. The release of interleukin-6 (IL-6) and tumor necrosis factor $\alpha(\mathrm{TNF} \alpha)$ was measured by an ELISA kit according to the manufacturer's instruction (Neobioscience Technology Co.,
Ltd., China). The detection limits are IL-6 $3.9 \mathrm{pg} \mathrm{mL} \mathrm{m}^{-1}$ and $\mathrm{TNF} \alpha 7.8 \mathrm{pg} \mathrm{mL}^{-1}$. The concentrations of cytokines in all the samples were higher than the detection limits.

\subsection{THP-1 adhesion}

The adhesion of THP-1 monocytes to HUVECs was performed as previously described. ${ }^{25}$ Briefly, HUVECs on 96-well black plates were exposed to $64 \mu \mathrm{g} \mathrm{mL} \mathrm{mL}^{-1}$ XFM19 or XFM21 either preincubated or not pre-incubated with BSA in serum free medium for $6 \mathrm{~h}$. THP-1 monocytes were labeled with $10 \mu \mathrm{M}$ CellTracker ${ }^{\mathrm{TM}}$ Green CMFDA (5-chloromethylfluorescein diacetate, Invitrogen, Carlsbad, CA), and $5 \times 10^{4}$ per well labeled THP-1 cells were incubated with the exposed HUVECs for another $1 \mathrm{~h}$ for adhesion. After that, the unbound THP-1 cells were washed away, and the green fluorescence from the adherent THP-1 cells was read at Ex $485 \pm 20 \mathrm{~nm}$ and Em $528 \pm$ $20 \mathrm{~nm}$ by an ELISA reader.

\subsection{Statistics}

All the data were expressed as means \pm S.E. (standard error) of means of four to five independent experiments $(n=3)$. Two-way ANOVA followed by a Tukey HSD test were used to compare the difference by using $\mathrm{R}$ 3.3.3. The $p$ value $<0.05$ was considered as statistically significantly different.

\section{Results}

\subsection{The topography of MWCNTs}

The topography of two types of MWCNTs, with or without preincubation with BSA, was investigated by AFM. As shown in Fig. 1A, both XFM19 and XFM21 showed a smooth surface when they were not pre-incubated with BSA. With the incubation with BSA, the surface of both types of MWCNTs became relatively coarse. The diameter of MWCNTs was increased after incubation with BSA (Fig. 1B).

\subsection{Hydrodynamic size and zeta potential of MWCNTs}

The distribution of hydrodynamic size and zeta potential of both types of MWCNTs with or without pre-incubation with BSA is shown in Fig. 2. Without pre-incubation with BSA, the average hydrodynamic sizes of XFM19 and XFM22 are about $245 \mathrm{~nm}$ and $210 \mathrm{~nm}$, respectively. With the pre-incubation with BSA, the 


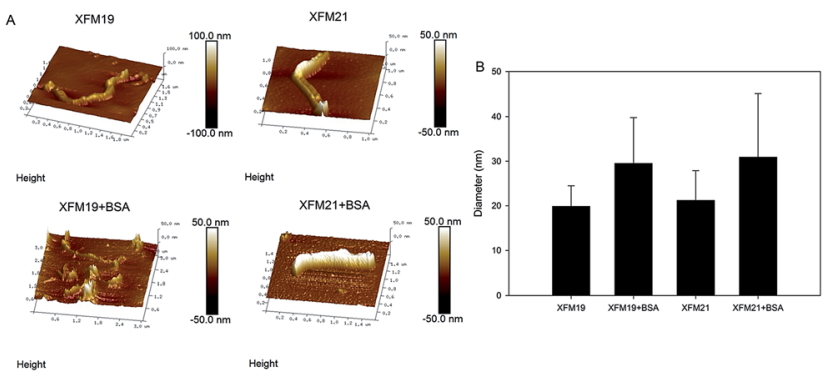

Fig. 1 The topographic changes of XFM19 (pristine MWCNT) and XFM21 (carboxylated MWCNT) with or without pre-incubation of BSA. (A) The representative images showing the topographic changes of MWCNTs. (B) The changes of MWCNT diameters due to the incubation with BSA. Data represented mean \pm SD of 50 randomly selected MWCNTS.

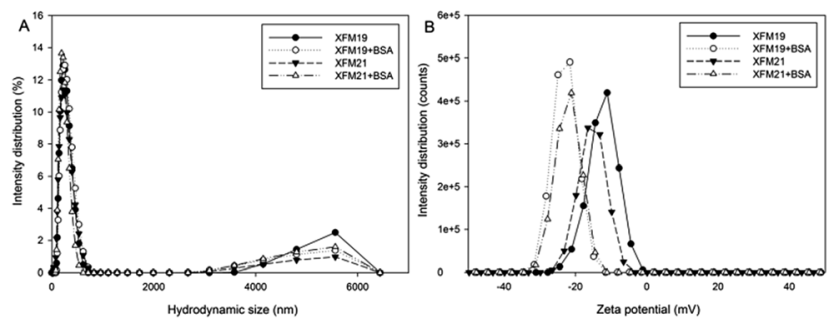

Fig. 2 The hydrodynamic size (A) and zeta potential (B) distribution of XFM19 (pristine MWCNT) and XFM21 (carboxylated MWCNT) with or without pre-incubation with BSA. A total of $640 \mu \mathrm{g} \mathrm{mL}^{-1}$ XFM19 or XFM21 was incubated with Milli-Q water or $5 \%$ BSA for 30 min, and then diluted to $16 \mu \mathrm{g} \mathrm{mL} \mathrm{mL}^{-1}$ XFM19 or XFM21 with or without the presence of $0.125 \%$ BSA. Data are representative of three measurements.

hydrodynamic sizes of both MWCNTs were not obviously changed. Both types of MWCNTs have a negative zeta potential, and the pre-incubation with BSA further decreased the zeta potential of both types of MWCNTs (Table 2).

\subsection{Internalization of MWCNTs}

The internalization of MWCNTs was observed by TEM and representative images are shown in Fig. 3. Without preincubation with BSA, the internalization of XFM19 (Fig. 3A) and XFM21 (Fig. 3C) could be observed, which appeared to be freely distributed in cytosol. With the pre-incubation with BSA, there appeared to be more internalization of XFM19 (Fig. 3B) and XFM21 (Fig. 3D).

\subsection{Cytotoxicity}

The cytotoxicity of MWCNTs, with or without the pre-incubation with BSA, to HUVECs is shown in Fig. 4. For the CCK-8 assay (Fig. 4A), exposure to $64 \mu \mathrm{g} \mathrm{mL}{ }^{-1}$ XFM19 or XFM21 without the pre-incubation with BSA was associated with significantly decreased cellular viability $(p<0.01)$. After pre-incubation with BSA, only XFM19 $(p<0.05)$, but not XFM21 $(p>0.05)$, showed significantly decreased cellular viability. Meanwhile, the cellular viability of HUVECs after exposure to $64 \mu \mathrm{g} \mathrm{mL}^{-1}$ XFM19 $(p<0.05)$ or XFM21 $(p<0.01)$ pre-incubated with BSA was significantly higher than that induced by the exposure to MWCNTs without pre-incubation with BSA.

For neutral red uptake assay, no significantly decreased neutral red uptake was observed after exposure to XFM19 or XFM21 with or without the pre-incubation with BSA $(p>0.05$; Fig. 4B).

\subsection{Oxidative stress}

As shown in Fig. 5A, exposure to XFM19 $(p<0.05)$ or XFM21 ( $p<$ 0.01 ) not pre-incubated with BSA significantly decreased the intracellular GSH. Meanwhile, the intracellular GSH in HUVECs after exposure to XFM21 pre-incubated with BSA was significantly higher than that in HUVECs after exposure to XFM21 not pre-incubated with BSA $(p<0.01)$.

For intracellular ROS (Fig. 5B), exposure to both types of MWCNTs with or without pre-incubation with BSA was associated with significantly increased intracellular ROS in HUVECs $(p<0.01)$.

\subsection{The release of IL- 6 and TNF $\alpha$}

The release of IL- 6 and TNF $\alpha$ is shown in Fig. 6. For the release of IL-6 (Fig. 6A), significantly increased IL-6 release was only observed after exposure to XFM19 or XFM21 pre-incubated with BSA $(p<0.01)$. The release of IL-6 in HUVECs after exposure to both types of MWCNTs pre-incubated with BSA was significantly higher than that induced by the exposure of MWCNTs not pre-incubated with BSA $(p<0.01)$. For the release of TNF $\alpha$ (Fig. 6B), BSA or XFM19 pre-incubated with BSA significantly promoted the release of TNF $\alpha(p<0.05)$. In contrast, exposure to XFM21 did not significantly affect the release of TNF $\alpha(p>0.05)$.

\subsection{THP-1 adhesion}

As shown in Fig. 7, exposure to both types of MWCNTs with or without the pre-incubation with BSA significantly promoted THP-1 adhesion to HUVECs $(p<0.01)$.

Table 2 The average hydrodynamic sizes and zeta potential of XFM19 (pristine MWCNT) and XFM21 (carboxylated MWCNT) with or without preincubation with BSA

\begin{tabular}{|c|c|c|c|c|}
\hline & \multicolumn{2}{|c|}{ Hydrodynamic size (nm) } & \multicolumn{2}{|l|}{ Zeta potential (mV) } \\
\hline $16 \mu \mathrm{g} \mathrm{mL}{ }^{-1}$ XFM21 & $210.3 \pm 1.5$ & $204.0 \pm 1.7$ & $16 \mu \mathrm{g} \mathrm{mL}{ }^{-1}$ XFM21 & $210.3 \pm 1.5$ \\
\hline
\end{tabular}



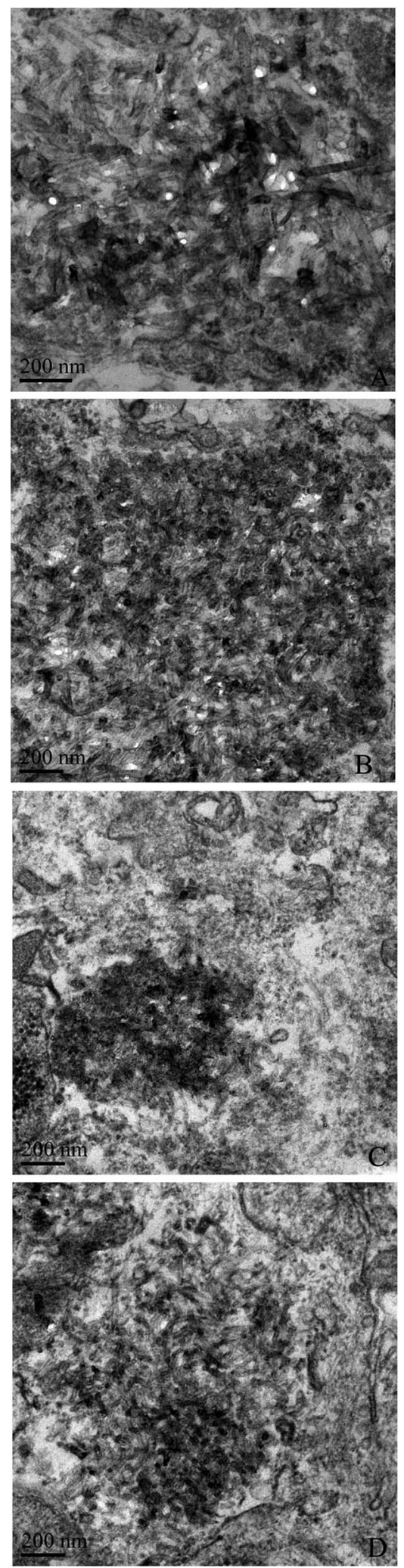

Fig. 3 TEM images of MWCNT exposed HUVECs. XFM19 (pristine MWCNT) and XFM21 (carboxylated MWCNT) were first incubated with Milli-Q water or $5 \%$ BSA for 30 min, and HUVECs were exposed to MWCNTs in serum free medium for $6 \mathrm{~h}$. TEM was used to indicate the internalization of MWCNTs. (A) The internalization of XFM19 into HUVECs. (B) The internalization of XFM19 pre-incubated with BSA into HUVECs. (C) The internalization of XFM21 into HUVECs. (D) The internalization of XFM21 pre-incubated with BSA into HUVECs.

\section{Discussion}

The blood is rich in proteins, therefore investigation of adverse health effects of NPs to ECs should consider the interactions between proteins and NPs. ${ }^{5,6}$ In this study, we investigated the influence of pre-incubation with BSA on HUVEC-MWCNT interactions. It was shown that both pristine and carboxylated MWCNTs were covered by BSA, which in turn led to increased diameters and decreased zeta potential of MWCNTs. MWCNTs were internalized into HUVECs, and the internalization was increased after MWCNTs were pre-incubated with BSA. Preincubation with BSA reduced the MWCNT induced cytotoxicity and depletion of GSH. However, MWCNT induced inflammatory response in HUVECs were increased after preincubation with BSA.

We first used AFM to indicate the topographic changes of MWCNTs due to the pre-incubation with BSA. After preincubation with BSA, the surfaces of both types of MWCNTs became relatively coarse (Fig. 1A). Moreover, the diameters of MWCNTs were increased after pre-incubation with BSA (Fig. 1B). In combination, these results indicated the formation of a protein corona. This is generally in agreement with previous studies showing the formation of protein corona onto carbonaceous NPs by AFM. ${ }^{26-28}$ Some studies indicated that the surface functionalization could influence the formation of the protein corona on MWCNTs. For example, it has been previously shown that more proteins were adsorbed onto the surface of carboxylated CNTs compared with the pristine ones..$^{15}$ In another study, it was shown that more proteins were adsorbed onto the surface of NPs if the proteins were modified to generate an additional carboxyl group.$^{29}$ However, in this study we found that the diameters of both pristine and carboxylated MWCNTs were equally increased following pre-incubation with BSA (Fig. 1B). In addition, pre-incubation with BSA did not obviously change the hydrodynamic size, but decreased the zeta potential of both types of MWCNTs (Fig. 2 and Table 2). Previous studies showed similar effects that the formation of a protein corona could influence the zeta potential of NPs. ${ }^{16,30,31}$ Thus, the data from this study might indicate that pre-incubation with BSA could influence the diameter and surface charge of MWCNTs regardless of the surface functionalization of NPs.

We then used TEM to visualize the internalization of MWCNTs. Recently, we have also used TEM to visualize the internalization of XFM19 into HUVECs, and it was shown that MWCNTs were located primarily in the nucleus and mitochondria. ${ }^{32}$ However, in this study it appeared that both types of MWCNTs were free in cytosol (Fig. 2). The differences in the internalization of MWCNTs in this study and our previous study could be due to the relatively shorter exposure time and the use of serum free conditions used in this study. Interestingly, after pre-incubation with BSA the internalization of MWCNTs was increased (Fig. 2). Some studies have also shown that the uptake of NPs could be increased when NPs were coated with proteins. For example, Yallapu et al. found improved internalization of magnetic NPs by cancer cells upon incubation with human serum. ${ }^{31}$ Similarly, Di Silvio et al. also observed increased 

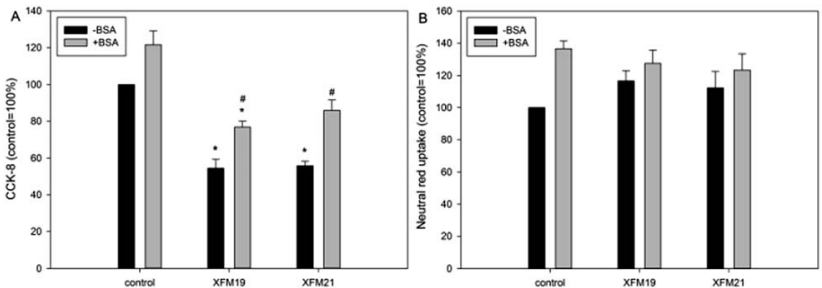

Fig. 4 Cytotoxicity as assessed by CCK-8 (A) or neutral red uptake assays (B). HUVECs were exposed to $64 \mu \mathrm{g} \mathrm{mL}{ }^{-1}$ XFM19 (pristine MWCNT) or XFM21 (carboxylated MWCNT) with or without pre-incubation with BSA in serum free medium for $6 \mathrm{~h}$, followed by CCK- 8 and neutral red uptake assays to indicate the cytotoxicity. *: $p<0.05$, compared with control; \#: $p<0.05$, compared between the groups exposed to MWCNTs with or without pre-incubation with BSA.

internalization of magnetic NPs into Caco-2 cells after incubation with proteins. ${ }^{33}$ In another study, it was shown that interactions with heparin enhanced the uptake of NPs into macrophages..$^{34}$ However, it should be noted that uptake of NPs could also be reduced by incubation with proteins. For instance, Cheng et al. found that protein corona inhibited the internalization of Au NPs by both phagocytic and nonphagocytic cells. ${ }^{35}$ Shannahan et al. showed that the formation of a protein corona reduced the internalization, and consequently the toxicity of Ag NPs to rat epithelial and endothelial cells. ${ }^{36}$ Therefore, the influence of the protein corona on NP internalization could be dependent on the types of NPs and cells and thus should be evaluated case by case.

The cytotoxicity assays showed that exposure to both types of MWCNTs was associated with decreased cellular viability, but not neutral red uptake. With the pre-incubation with BSA, the cellular viability of HUVECs after both types of MWCNT exposure increased (Fig. 4). This is generally in agreement with previous observations showing that the protein corona could mitigate the toxicity of CNTs. ${ }^{13,37}$ It has been suggested that MWCNT induced oxidative stress played an important role in the cytotoxicity. ${ }^{7}$ To this end we measured the intracellular GSH and ROS to indicate the oxidative stress. It was shown that the pre-incubation with BSA significantly increased the intracellular GSH after MWCNT exposure, whereas MWCNT induced ROS

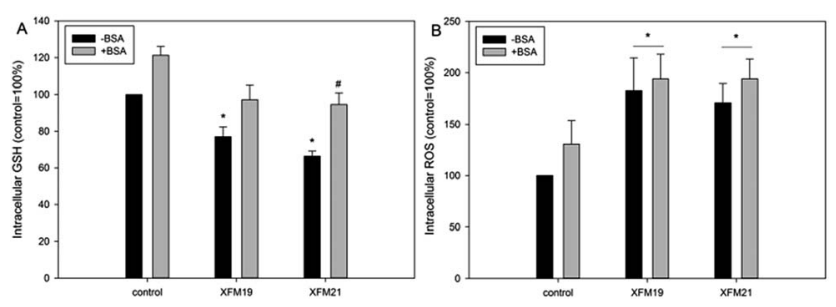

Fig. 5 Oxidative stress as assessed by the measurement of intracellular GSH (A) or ROS (B). HUVECs were exposed to $64 \mu \mathrm{g} \mathrm{mL}^{-1}$ XFM19 (pristine MWCNT) and XFM21 (carboxylated MWCNT) with or without pre-incubation with BSA in serum free medium for $6 \mathrm{~h}$, followed by the measurement of intracellular GSH or ROS to indicate oxidative stress. *: $p<0.05$, compared with control; \#: $p<0.01$, compared between the groups exposed to MWCNTs with or without pre-incubation with BSA.

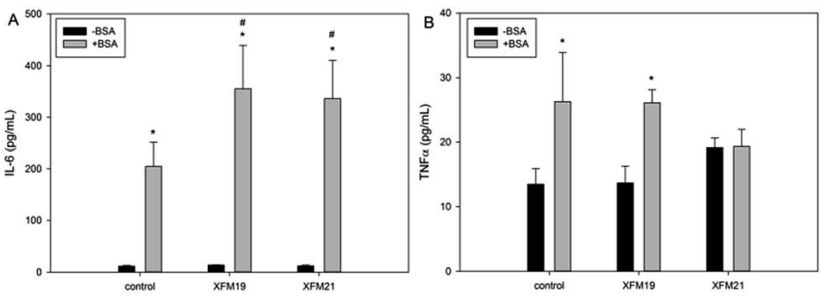

Fig. 6 The release of IL- 6 (A) and TNF $\alpha$ (B). HUVECs were exposed to $64 \mu \mathrm{g} \mathrm{mL}{ }^{-1}$ XFM19 (pristine MWCNT) and XFM21 (carboxylated MWCNT) with or without pre-incubation with BSA in serum free medium for $6 \mathrm{~h}$. The supernatants were collected and centrifuged prior to the measurement of IL- 6 or TNF $\alpha$ by ELISA. *: $p<0.01$, compared with control; \#: $p<0.01$, compared between the groups exposed to MWCNTs with or without pre-incubation with BSA.

was not further influenced by BSA pre-incubation (Fig. 5). These data combined suggested that BSA could reduce MWCNT induced oxidative stress by preventing GSH depletion. Previously, it has been shown that the protein corona reduced NP induced oxidative stress by reduction of the NP oxidative potential or alteration of the anti-oxidant signaling pathway. ${ }^{38-40}$ The data obtained in this study are consistent with previous reports, and the fact that BSA reduced MWCNT induced cytotoxicity could be explained by the fact that protein corona formation significantly alleviated NP induced GSH depletion.

It was recently shown that the coating of the protein corona could alter the immunological identity of NPs..$^{41}$ For example, Kurtz-Chalot et al. ${ }^{42}$ showed surface-functionalization dependent protein corona formation on silica NPs, which consequently influenced the release of TNF $\alpha$ from macrophages. Similarly, Di Cristo et $a l^{43}$ showed that pyrogenic silica NPs interacted with more proteins and thus elicited a larger inflammatory response in murine macrophages compared with precipitated NPs. In another study, Dai et al. ${ }^{44}$ found that protein corona formation on an engineered NP surface deeply influenced NP induced inflammatory responses in human

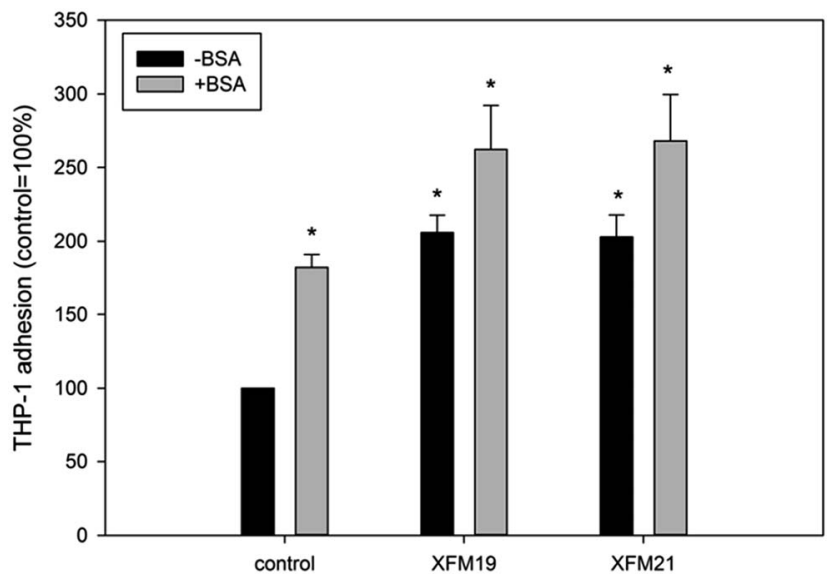

Fig. 7 The THP-1 adhesion to HUVECs. HUVECs were exposed to $64 \mu \mathrm{g} \mathrm{mL}^{-1}$ XFM19 (pristine MWCNT) and XFM21 (carboxylated MWCNT) with or without pre-incubation with BSA in serum free medium for $6 \mathrm{~h}$, followed by the adhesion assay. *: $p<0.01$, compared with control. 
monocytes. However, most of the studies in this area used professional immune cells such as macrophages, and the immune responses induced by protein coated NPs to other types of cells are ignored. ECs play an important role in the regulation of blood vessel function, and exposure to NPs has been shown to induce inflammatory responses of ECs. ${ }^{5,9}$ Results from the present study showed that IL- 6 was only induced after exposure to BSA pre-incubated MWCNTs (Fig. 6), which suggested that protein corona formation on MWCNTs might amplify the inflammatory responses. Similar observations were reported recently, that protein corona promoted NPs induced inflammatory responses, although the researchers used macrophages rather than ECs. ${ }^{17,43}$

The adhesion of monocyte to ECs and consequently the transformation of monocytes into macrophages is a hallmark of atherosclerosis. ${ }^{5,9}$ In this study, we quantified the adhesion of monocytes to HUVECs, and the results showed that monocyte adhesion could be induced by both types of HUVECs (Fig. 7). The increased adhesion of monocytes to HUVECs after CNT exposure has also been reported before. ${ }^{25,45,46}$ However, to the best of our knowledge, no previous study investigated the influence of protein corona formation on NP induced monocyte adhesion. In this study, it was shown that monocyte adhesion could be significantly induced by MWCNTs with or without preincubation with BSA, which indicated that the protein corona might not be important in determining the adhesion. MWCNT induced IL-6 release was enhanced after pre-incubation with BSA (Fig. 6), but it has been shown before that IL-6 release is not correlated with MWCNT induced monocyte adhesion to HUVECs. ${ }^{25}$

\section{Conclusions}

In summary, the results from this study indicated that both pristine and carboxylated MWCNTs could be covered by BSA, which led to the change of diameter and zeta potential of MWCNTs. Pre-incubation with BSA increased the internalization of MWCNTs into HUVECs. This in turn led to reduced cytotoxicity of MWCNTs to HUVECs, associated with reduced oxidative stress. However, the inflammatory responses were enhanced when the cells were exposed to MWCNTs preincubated with BSA. Since the presence of BSA also enhanced the cellular viability, release of inflammatory cytokines and monocyte adhesion, these alterations are likely to be due to a combined effect of both MWCNTs and BSA. The data from this study might suggest that when assessing the toxicity of NPs to ECs, it is necessary to consider the interactions between NPs and proteins present in the blood.

\section{Conflicts of interest}

There are no conflicts to declare.

\section{Acknowledgements}

This work was supported by the National Natural Science Foundation of China (no. 31660729 \& 21704104).

\section{Notes and references}

1 M. F. De Volder, S. H. Tawfick, R. H. Baughman and A. J. Hart, Science, 2013, 339, 535.

2 T. Kuang, L. Chang, F. Chen, Y. Sheng, D. Fu and X. Peng, Carbon, 2016, 105, 305.

3 Y. Zhang, D. Petibone, Y. Xu, M. Mahmood, A. Karmakar, D. Casciano, S. Ali and A. S. Biris, Drug Metab. Rev., 2014, 46, 232.

4 B. S. Wong, S. L. Yoong, A. Jagusiak, T. Panczyk, H. K. Ho, W. H. Ang and G. Pastorin, Adv. Drug Delivery Rev., 2013, 65, 1964.

5 Y. Cao, Y. Gong, L. Liu, Y. Zhou, X. Fang, C. Zhang, Y. Li and J. Li, J. Appl. Toxicol., 2017, 37, 1359.

6 M. I. Setyawati, C. Y. Tay, D. Docter, R. H. Stauber and D. T. Leong, Chem. Soc. Rev., 2015, 44, 8174.

7 P. Moller, D. V. Christophersen, D. M. Jensen, A. Kermanizadeh, M. Roursgaard, N. R. Jacobsen, J. G. Hemmingsen, P. H. Danielsen, Y. Cao, K. Jantzen, H. Klingberg, L. G. Hersoug and S. Loft, Arch. Toxicol., 2014, 88, 1939.

8 P. Moller, D. V. Christophersen, N. R. Jacobsen, A. Skovmand, A. C. Gouveia, M. H. Andersen, A. Kermanizadeh, D. M. Jensen, P. H. Danielsen, M. Roursgaard, K. Jantzen and S. Loft, Crit. Rev. Toxicol., 2016, 46, 437.

9 Y. Cao, J. Long, Y. Ji, G. Chen, Y. Shen, Y. Gong and J. Li, Inhalation Toxicol., 2016, 28, 583.

10 K. A. Tomaszewski, M. W. Radomski and M. J. SantosMartinez, Nanomedicine, 2015, 10, 1451.

11 D. Docter, D. Westmeier, M. Markiewicz, S. Stolte, S. K. Knauer and R. H. Stauber, Chem. Soc. Rev., 2015, 44, 6094.

12 C. Ge, J. Tian, Y. Zhao, C. Chen, R. Zhou and Z. Chai, Arch. Toxicol., 2015, 89, 519.

13 K. Bhattacharya, S. P. Mukherjee, A. Gallud, S. C. Burkert, S. Bistarelli, S. Bellucci, M. Bottini, A. Star and B. Fadeel, Nanomedicine, 2016, 12, 333.

14 X. Cai, R. Ramalingam, H. S. Wong, J. Cheng, P. Ajuh, S. H. Cheng and Y. W. Lam, Nanomedicine, 2013, 9, 583.

15 J. H. Shannahan, J. M. Brown, R. Chen, P. C. Ke, X. Lai, S. Mitra and F. A. Witzmann, Small, 2013, 9, 2171.

16 X. Zhao, D. Lu, F. Hao and R. Liu, J. Hazard. Mater., 2015, 292, 98 .

17 W. Bai, A. Raghavendra, R. Podila and J. M. Brown, Int. J. Nanomed., 2016, 11, 4357.

18 S. H. De Paoli, L. L. Diduch, T. Z. Tegegn, M. Orecna, M. B. Strader, E. Karnaukhova, J. E. Bonevich, K. Holada and J. Simak, Biomaterials, 2014, 35, 6182.

19 Y. Ji, M. Zhu, Y. Gong, H. Tang, J. Li and Y. Cao, Basic Clin. Pharmacol. Toxicol., 2017, 120, 79.

20 Q. Jiang, X. Li, S. Cheng, Y. Gu, G. Chen, Y. Shen, Y. Xie and Y. Cao, Environ. Toxicol. Pharmacol., 2016, 48, 103.

21 G. Repetto, P. A. del and J. L. Zurita, Nat. Protoc., 2008, 3, 1125.

22 J. C. Fernandez-Checa and N. Kaplowitz, Anal. Biochem., 1990, 190, 212. 
23 Y. Cao, K. Jantzen, A. C. Gouveia, A. Skovmand, M. Roursgaard, S. Loft and P. Moller, Environ. Toxicol. Pharmacol., 2015, 40, 164.

24 Y. Shen, G. Chen, A. Xiao, Y. Xie, L. Liu and Y. Cao, In vitro effect of flaxseed oil and alpha-linolenic acid against the toxicity of lipopolysaccharide (LPS) to human umbilical vein endothelial cells, Inflammopharmacology, 2017, DOI: 10.1007/s10787-017-0400-x.

25 Y. Cao, M. Roursgaard, N. R. Jacobsen, P. Moller and S. Loft, Nanotoxicology, 2016, 10, 235.

26 G. Duan, S. G. Kang, X. Tian, J. A. Garate, L. Zhao, C. Ge and R. Zhou, Nanoscale, 2015, 7, 15214.

27 W. Hu, C. Peng, M. Lv, X. Li, Y. Zhang, N. Chen, C. Fan and Q. Huang, ACS Nano, 2011, 5, 3693.

28 C. Ge, J. Du, L. Zhao, L. Wang, Y. Liu, D. Li, Y. Yang, R. Zhou, Y. Zhao, Z. Chai and C. Chen, Proc. Natl. Acad. Sci. U. S. A., 2011, 108, 16968.

29 L. Treuel, S. Brandholt, P. Maffre, S. Wiegele, L. Shang and G. U. Nienhaus, ACS Nano, 2014, 8, 503.

30 S. Tenzer, D. Docter, J. Kuharev, A. Musyanovych, V. Fetz, R. Hecht, F. Schlenk, D. Fischer, K. Kiouptsi, C. Reinhardt, K. Landfester, H. Schild, M. Maskos, S. K. Knauer and R. H. Stauber, Nat. Nanotechnol., 2013, 8, 772.

31 M. M. Yallapu, N. Chauhan, S. F. Othman, V. KhalilzadSharghi, M. C. Ebeling, S. Khan, M. Jaggi and S. C. Chauhan, Biomaterials, 2015, 46, 1.

32 J. Long, Y. Xiao, L. Liu and Y. Cao, J. Nanobiotechnol., 2017, 15, 80.

33 S. D. Di, N. Rigby, B. Bajka, A. Mackie and B. F. Baldelli, Int. J. Biochem. Cell Biol., 2016, 75, 212.

34 S. Schottler, K. Klein, K. Landfester and V. Mailander, Nanoscale, 2016, 8, 5526.
35 X. Cheng, X. Tian, A. Wu, J. Li, J. Tian, Y. Chong, Z. Chai, Y. Zhao, C. Chen and C. Ge, ACS Appl. Mater. Interfaces, 2015, 7, 20568.

36 J. H. Shannahan, R. Podila, A. A. Aldossari, H. Emerson, B. A. Powell, P. C. Ke, A. M. Rao and J. M. Brown, Toxicol. Sci., 2015, 143, 136.

37 S. Lanone, P. Andujar, A. Kermanizadeh and J. Boczkowski, Adv. Drug Delivery Rev., 2013, 65, 2063.

38 S. Vranic, I. Gosens, N. R. Jacobsen, K. A. Jensen, B. Bokkers, A. Kermanizadeh, V. Stone, A. Baeza-Squiban, F. R. Cassee, L. Tran and S. Boland, Arch. Toxicol., 2017, 91, 353.

39 R. Zukiene and V. Snitka, Colloids Surf., B, 2015, 135, 316.

40 P. Chandran, J. E. Riviere and N. A. Monteiro-Riviere, Nanotoxicology, 2017, 11, 507.

41 M. Neagu, Z. Piperigkou, K. Karamanou, A. B. Engin, A. O. Docea, C. Constantin, C. Negrei, D. Nikitovic and A. Tsatsakis, Arch. Toxicol., 2017, 91, 1031.

42 A. Kurtz-Chalot, C. Villiers, J. Pourchez, D. Boudard, M. Martini, P. N. Marche, M. Cottier and V. Forest, Mater. Sci. Eng., C, 2017, 75, 16.

43 C. L. Di, D. Movia, M. G. Bianchi, M. Allegri, B. M. Mohamed, A. P. Bell, C. Moore, S. Pinelli, K. Rasmussen, J. Riego-Sintes, A. Prina-Mello, O. Bussolati and E. Bergamaschi, Toxicol. Sci., 2016, 150, 40.

44 Q. Dai, J. Guo, Y. Yan, C. S. Ang, N. Bertleff-Zieschang and F. Caruso, Biomacromolecules, 2017, 18, 431.

45 Y. Cao, N. R. Jacobsen, P. H. Danielsen, A. G. Lenz, T. Stoeger, S. Loft, H. Wallin, M. Roursgaard, L. Mikkelsen and P. Moller, Toxicol. Sci., 2014, 138, 104.

46 Y. Suzuki, S. Tada-Oikawa, Y. Hayashi, K. Izuoka, M. Kataoka, S. Ichikawa, W. Wu, C. Zong, G. Ichihara and S. Ichihara, Part. Fibre Toxicol., 2016, 13, 54. 